\title{
Hagamos que nuestra acción se multiplique
}

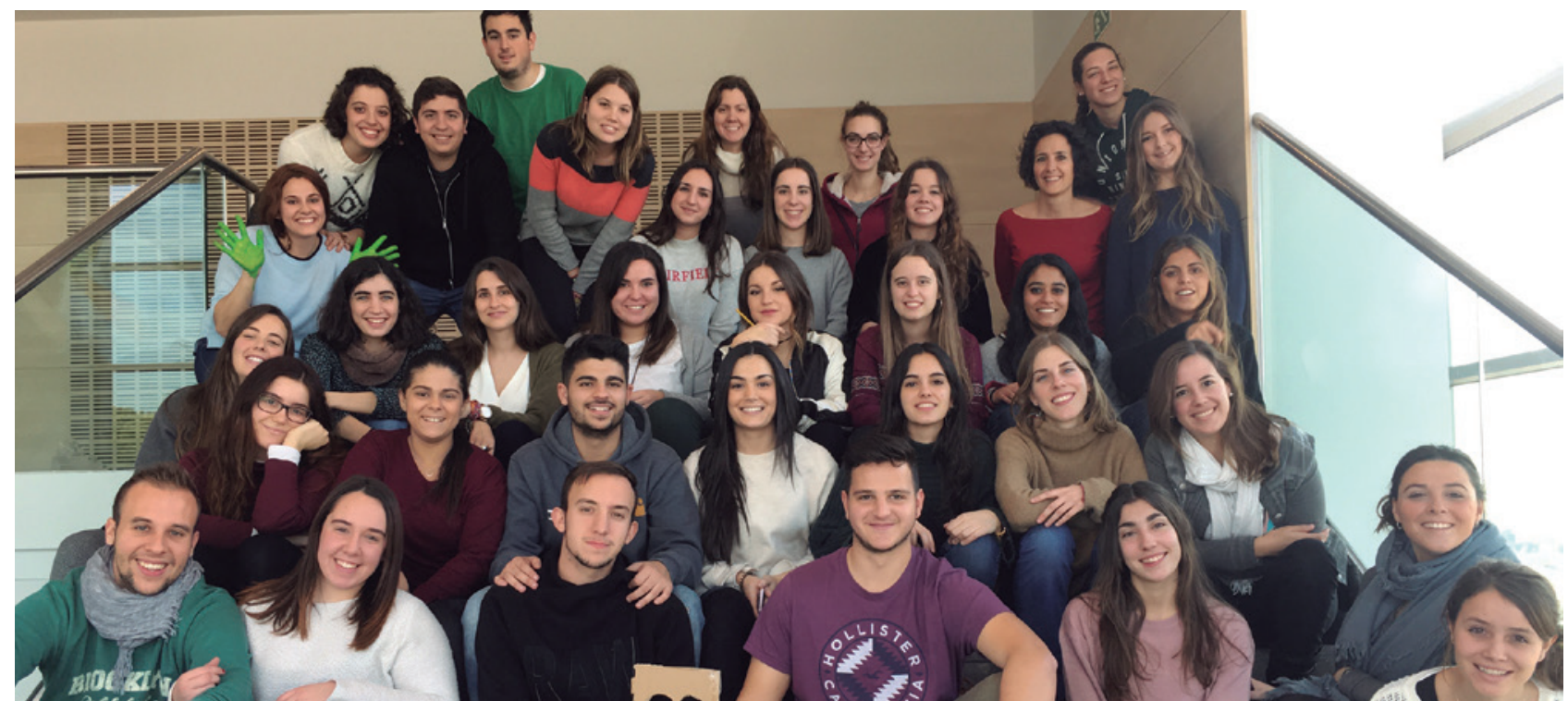

Movidos por nuestro interés en la educación, en la innovación didáctica y metodológica, y en el reparto equitativo de oportunidades en todos los ámbitos educativos, en la materia de Didáctica de las Matemáticas, decidimos incorporar una intervención pedagógica que aunara estos intereses y nos permitiera enriquecer nuestro proceso de formación como docentes. Así comenzó en el grupo de estudiantes de $3^{\circ}$ del grado de Educación Primaria de la Universidad Pontificia Comillas la andadura del Aprendizaje Servicio (ApS)

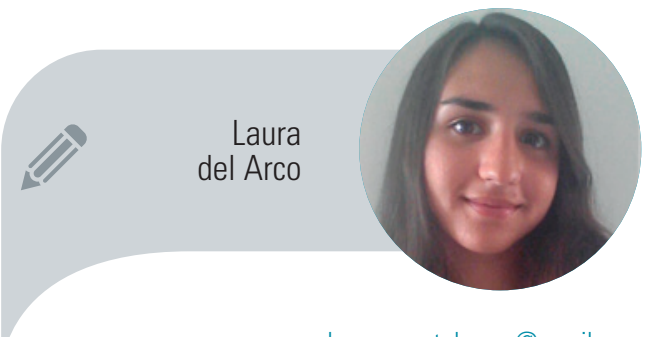
que ha conseguido vincular a Guatemala y a España con una excusa matemática: la enseñanza de la multiplicación. 


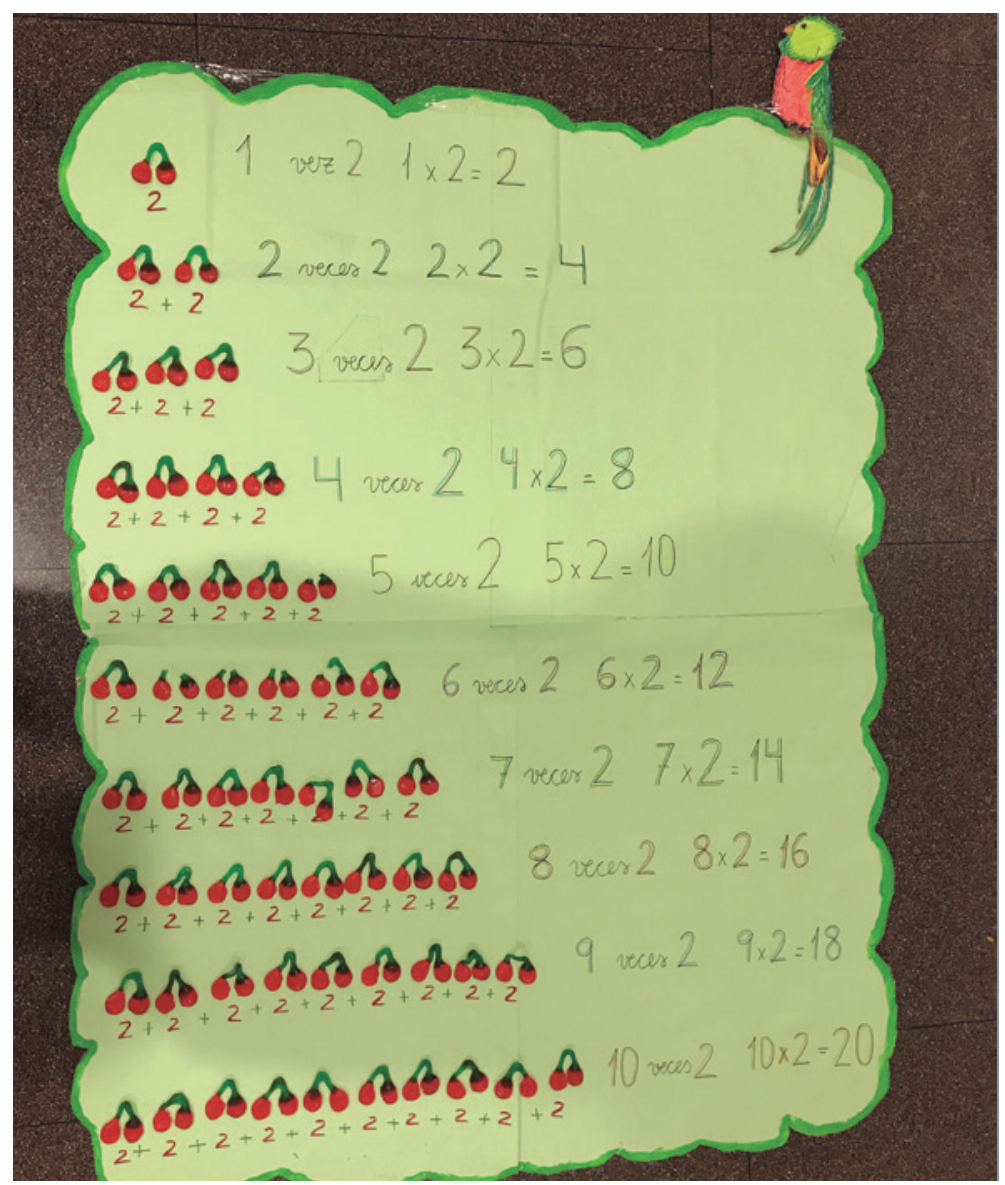

El servicio desinteresado ennoblece al hombre, lo dota de inteligencia y de las habilidades necesarias para refinar la naturaleza humana. Cumplir con el deber propio no es suficiente. Se deben cultivar el amor y la compasión.

(AnÓNIMO)

Nuestro nexo de unión ha sido la Fundación del Valle que partiendo de la premisa "educación, un camino para la paz", se encarga de llevar a cabo iniciativas sociales de carácter educativo tanto en el ámbito nacional como en el internacional. Desde el año 2001 promueve en Guatemala proyectos de alfabetización, de calidad educativa y de formación en derechos humanos. Quince años después, una clase formada por treinta y siete futuros maestros hemos querido contribuir en esta compleja labor.

\section{Guatemala}

Una alianza entre las profesoras de Didáctica de las Matemáticas (Elsa Santaolalla) y de Innovación Educativa (Tamara Díaz) propició que la Fundación del Valle dedicará una sesión formativa sobre la metodología del ApS, en la que también se habló del contexto concreto con el que íbamos a vincular nuestra acción.

Los números nos ayudaron a conocer Guatemala: un país con una superficie de $108.890 \mathrm{~km}^{2}$; dividido en 8 regiones, en el que aunque su idioma oficial es el español, se hablan 22 lenguas no oficiales. También los números nos permitieron acercarnos a su sistema educativo al que se destina un 2,8\% del PIB y en el que por término medio sus estudiantes sólo están escolarizados 5 años a pesar de que hay previstos 10

Sin embargo en esa jornada también descubrimos que Guatemala es mucho más que números. Guatemala son sus playas, sus monumentos, sus colores, su alegría y su forma de ver la vida. En definitiva, lo que hace de Guatemala un lugar mágico son sus personas y es a ellas a quienes debíamos y queríamos ayudar.

Nuestra labor en el proyecto era colaborar en la formación de 60 maestros de las escuelas rurales de las regiones del occidente de Guatemala para que pudiesen enseñar a sus alumnos de primaria cómo aprender a multiplicar de forma significativa. Nuestra misión estaba a punto de comenzar.

\section{Manos a la obra}

El primer paso fue documentarse en múltiples artículos y libros de Didáctica de las Matemáticas que abordaban la enseñanza y el aprendizaje de la multiplicación.

Presentar los conceptos desde distintos enfoques genera un aprendizaje significativo. Alsina (2016) propone para ello el empleo de la pirámide de la educación matemática, diagrama en cuya base se encuentran los recursos que el profesor de Matemáticas ha de utilizar diariamente, tales como situaciones cotidianas y matematización del entorno y, a medida que se asciende por el tronco de la pirámide, aparecen recursos que hay que usar esporádicamente, estando el libro de texto en la cúspide. Así, dado que el profesor ha de incorporar recursos procedentes del entorno y de la vida cotidiana para desarrollar la competencia matemática, 


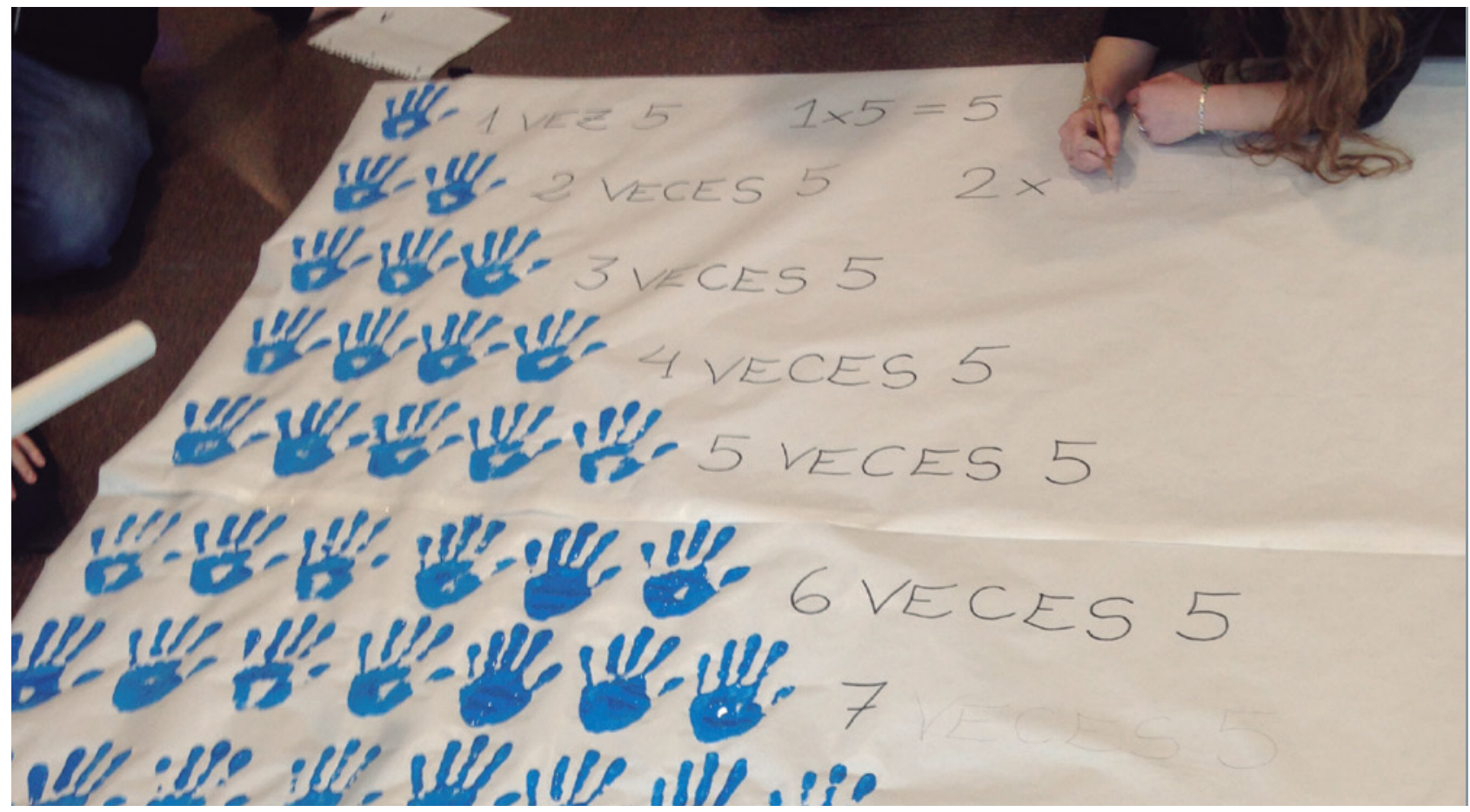

entendimos la necesidad de crear materiales manipulativos.

Estos recursos juegan un papel muy importante durante el aprendizaje de los conceptos, ya que, para entender las matemáticas, antes hay que vivirlas. Tal y como María Montessori asegura "el niño tiene la inteligencia en la mano", y por ello nuestro primer deber es brindarle la ocasión de operar con lobjetos concretos. Este proceso corresponde a la primera fase de la adquisición de los conceptos matemáticos, que es la manipulativa (Santaolalla, 2011). Para la consecución de este fin vamos a utilizar su entorno más cercano y lo vamos a matematizar. Es decir, si tomamos como ejemplo la flor nacional de Guatemala, la Monja Blanca, podemos emplear sus pétalos para abordar esta primera fase de la multiplicación. Este elemento natural y de la vida cotidiana posee siempre 3 pétalos, por lo que es un recurso ideal para familiarizarnos con la tabla del 3 de manera manipulativa. Así, si presentamos 2 flores se puede observar que cada una de ellas tiene 3 pétalos, por lo tanto, 2 veces 3, en total: 6 pétalos.

En la segunda fase, llamada simbólica, se inicia el paso de lo concreto a lo abstracto, de modo que el niño, además de manipular objetos concretos, ya puede comenzar a trabajar con representaciones gráficas y símbolos. Siguiendo con el ejemplo de la flor mencionado previa-

\section{A nivel profesional hemos oblenido muchos beneficios, entre los cuales se encuentra el descubrimiento de la propuesta educaliva del Aprendizaje Servicio, tan necesario en nuestra sociedad actual, y el enriquecimiento gracias a la interacción que fomenta el aprendizaje cooperalivo}

mente, en esta fase podríamos ofrecer a los alumnos una imagen con 2 Monjas Blancas. En este caso, ya no diríamos que cada flor tiene 3 pétalos, sino que se vería la representación de 2 veces 3, y se asociaría el concepto 'veces' a la multiplicación, sin introducir aún el algoritmo matemático que corresponde a la siguiente fase.

En la tercera y última fase, Ilamada de abstracción, se llega finalmente a los signos abstractos y arbitrarios y a los algoritmos. Por ello, Fernández Bravo (2007a) insiste en que es en este momento cuando deben asociar la palabra 'veces' al signo ' $x$ ' que se lee 'multiplicado por' y que de forma abreviada se dice 'por'.

En la imagen hemos representado el proceso completo en dos casos distintos, el 2x3 con el número de pétalos que reúnen dos flores típicas de Guatemala y el 3×4 para el número de ruedas que hay 

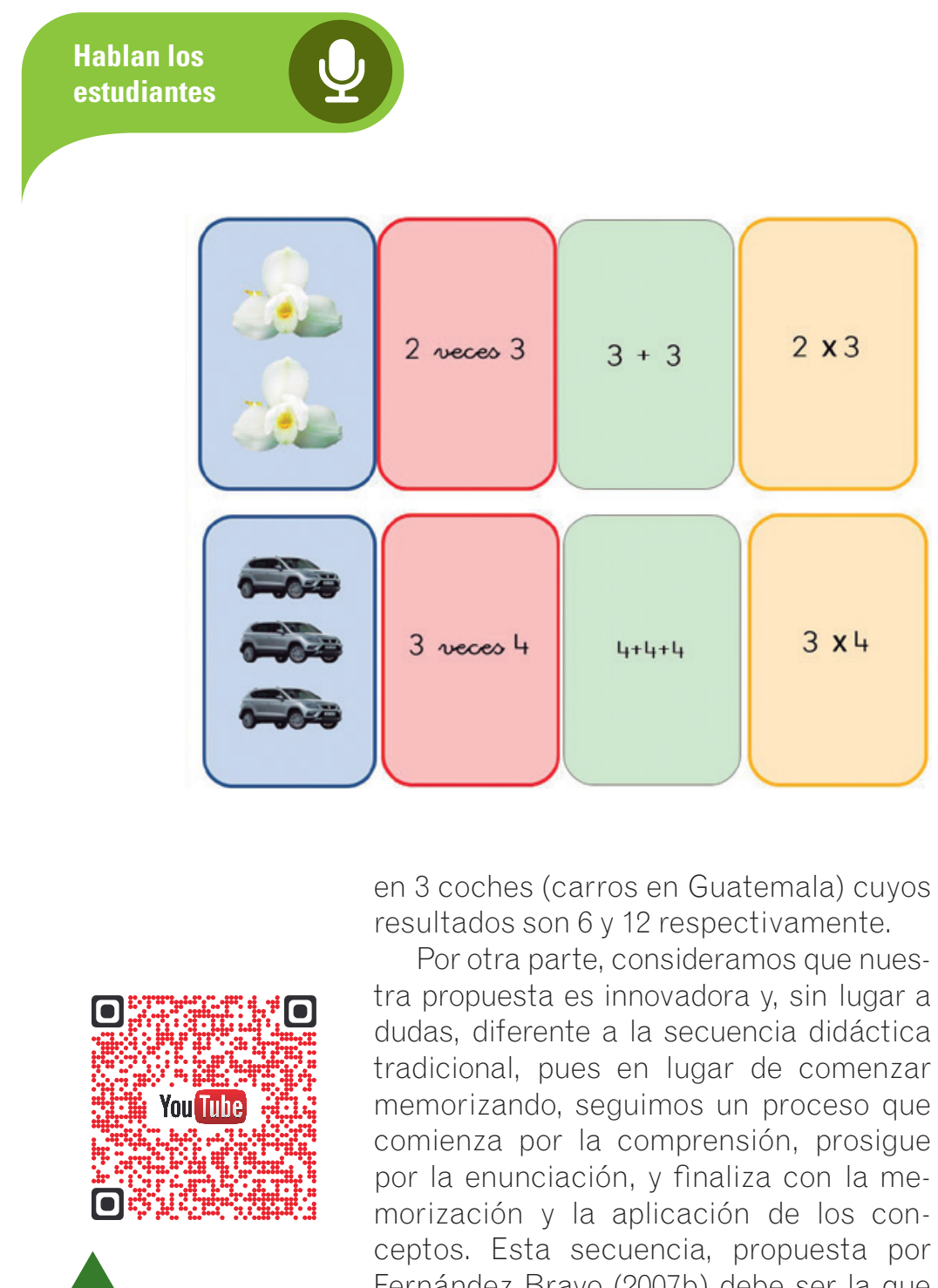

Nuestra acción se multiplica en 3 coches (carros en Guatemala) cuyos resultados son 6 y 12 respectivamente.

Por otra parte, consideramos que nuestra propuesta es innovadora y, sin lugar a dudas, diferente a la secuencia didáctica tradicional, pues en lugar de comenzar memorizando, seguimos un proceso que comienza por la comprensión, prosigue por la enunciación, y finaliza con la memorización y la aplicación de los conceptos. Esta secuencia, propuesta por Fernández Bravo (2007b) debe ser la que se utilice para presentar y aprender cualquier contenido matemático en las aulas. De este modo, los alumnos se convierten en los protagonistas de su propio aprendizaje apoyándose en el maestro-guía, pero siendo ellos mismos quienes construyen las tablas de multiplicar y acaban recitando rimas y canciones para favorecer la memorización de las tablas.

Respaldados por esta fundamentación teórica y siempre bajo la supervisión de nuestra profesora de Didáctica de las Matemáticas, los distintos grupos de trabajo cooperativo en los que dividimos la clase fuimos capaces de construir materiales y talleres variados que se ajustaban a las diversas fases del proceso de aprendizaje de la multiplicación.

\section{Silencio, se rueda}

Antes de guardar todos los materiales en cajas para enviarlas a su destino final, llegó el momento de las grabaciones. El formato digital resultó ser un aliado, no solamente para presentarnos a Guatemala y dar a conocer nuestros talleres y dinámicas, sino también para explicar los diversos juegos y las nociones teóricas que subyacían en los mismos. Así, con la ilusión y los nervios ondeando en el ambiente, las cámaras hicieron su aparición en nuestras aulas. ¡Tres, dos, uno y... acción!

El objetivo se fijó en los talleres diseñados con cartas: Memory, Cuadrado, Familia, Doubble, Parejas..., infinidad de juegos se podían realizar con las diferentes barajas de cartas creadas por los grupos. El tradicional Burro se transformó en un recurso lúdico, motivador y placentero para aprender a asociar las diferentes maneras de representar una multiplicación. De este modo, el objetivo de los jugadores era conseguir las cuatro cartas que hicieran referencia a la misma multiplicación (por ejemplo: 3 veces $8,8+8+8,3 \times 8,24$ ).

Luego, todos los focos se desviaron al Bingo Matemático, en el que la persona encargada del juego debía ir cantando los resultados de las multiplicaciones y, los jugadores, ir tachando las casillas de sus cartones. En estas casillas no aparecía sólo el resultado, sino también otras maneras de representar la multiplicación.

También las risas y el interés se multiplicaron cuando dos compañeros de clase tocaron la guitarra española y cantaron el famoso Bailando de Enrique Iglesias versionado. La nueva letra era un conjunto de rimas para facilitar la memorización y el aprendizaje de la tabla del dos. Multiplicando, que así se titula el nuevo éxito musical, no sólo es pegadiza, sino que constituye una fuente de aprendizaje divertido y atractivo para los niños. Podéis acceder a esta canción y a todos los vídeos escaneando el código QR.

Por último, también se presentaron las tablas de multiplicar que habíamos construido. Para que se pueda producir ese aprendizaje significativo y duradero, es preciso que los alumnos sean agentes activos del proceso educativo y se impliquen en la construcción de su propio conocimiento. Así, no hay que dar las tablas de multiplicar ya hechas, sino crearlas con los niños.

Emulando este proceso, los grupos cooperativos de la clase nos repartimos las diferentes tablas de multiplicar y cada grupo construyó una: la tabla del dos con 
cerezas, la tabla del cuatro con tenedores de cuatro puntas, la tabla del cinco con los dedos de una mano, la tabla del ocho con arañas ide gominola! o la tabla del diez utilizando las dos manos.

¿Por qué esos elementos y no otros? Porque las tablas se han creado con materiales procedentes de la vida cotidiana y que, por naturaleza, son objetos que vienen siempre de dos en dos (las cerezas), de cinco en cinco (los dedos de una mano) o de ocho en ocho (las patas de una araña). Estos grandes murales son maneras visuales, atractivas, sencillas y manipulativas para aprender las tablas de multiplicar.

Fue una jornada diferente, estimulante y gratificante en la que los estudiantes de $3^{\circ}$ del grado en Educación Primaria fuimos los actores aunque los protagonistas fueron siempre los niños de las escuelas rurales de Guatemala.

\section{Sentimientos multiplicados}

Cuando las cámaras se apagaron se encendió en nosotros una llama difícil de extinguir.

A lo largo de este proyecto hemos podido experimentar multitud de sentimientos. Al principio, un poco confusos, dudábamos de nuestra capacidad para poder lograrlo pero estábamos impacientes por ponernos manos a la obra. A medida que se fue desarrollando el proyecto, siempre tuvimos el apoyo y la guía de nuestros profesores de la Universidad. Esto hizo que esos primeros sentimientos se transformasen en optimismo y gratitud por la oportunidad que se nos brindaba.

Asimismo, a nivel profesional hemos obtenido muchos beneficios, entre los cuales se encuentra el descubrimiento de la propuesta educativa del Aprendizaje-Servicio, tan necesario en nuestra sociedad actual, y el enriquecimiento gracias a la interacción que fomenta el aprendizaje cooperativo. Además, nuestro bagaje cultural ha aumentado por el conocimiento de la realidad de otros países, así como por la investigación y profundización en el estudio del tema matemático de la multiplicación.

El proyecto también nos ha enseñado la importancia del esfuerzo, de

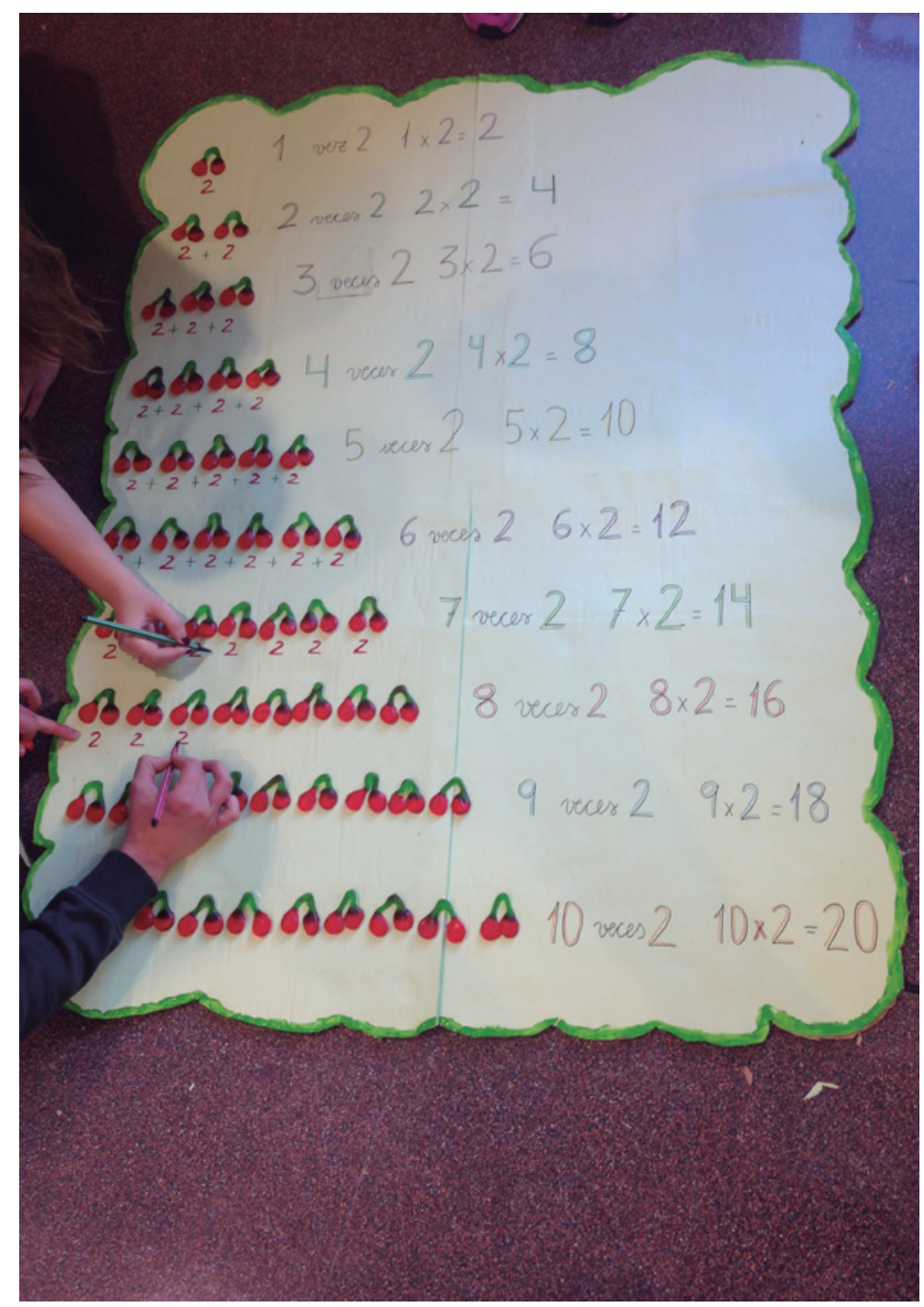

El ApS para Gualemala nos ha ayudado a

entender que la educación es una herramienta

más poderosa de lo que podemos imaginar,

pues con ella podemos cambiar el mundo

la superación y del trabajo, factores indispensables para fabricar recursos, buscar marcos teóricos o explicar los talleres ante las cámaras. Y, finalmente, y $\sin$ atisbo de duda, hemos obtenido un impulso para seguir innovando, aprendiendo y formándonos día a día. Pues, como afirmó John Cotton Dana "quien se atreva a enseñar, nunca debe dejar de aprender". 


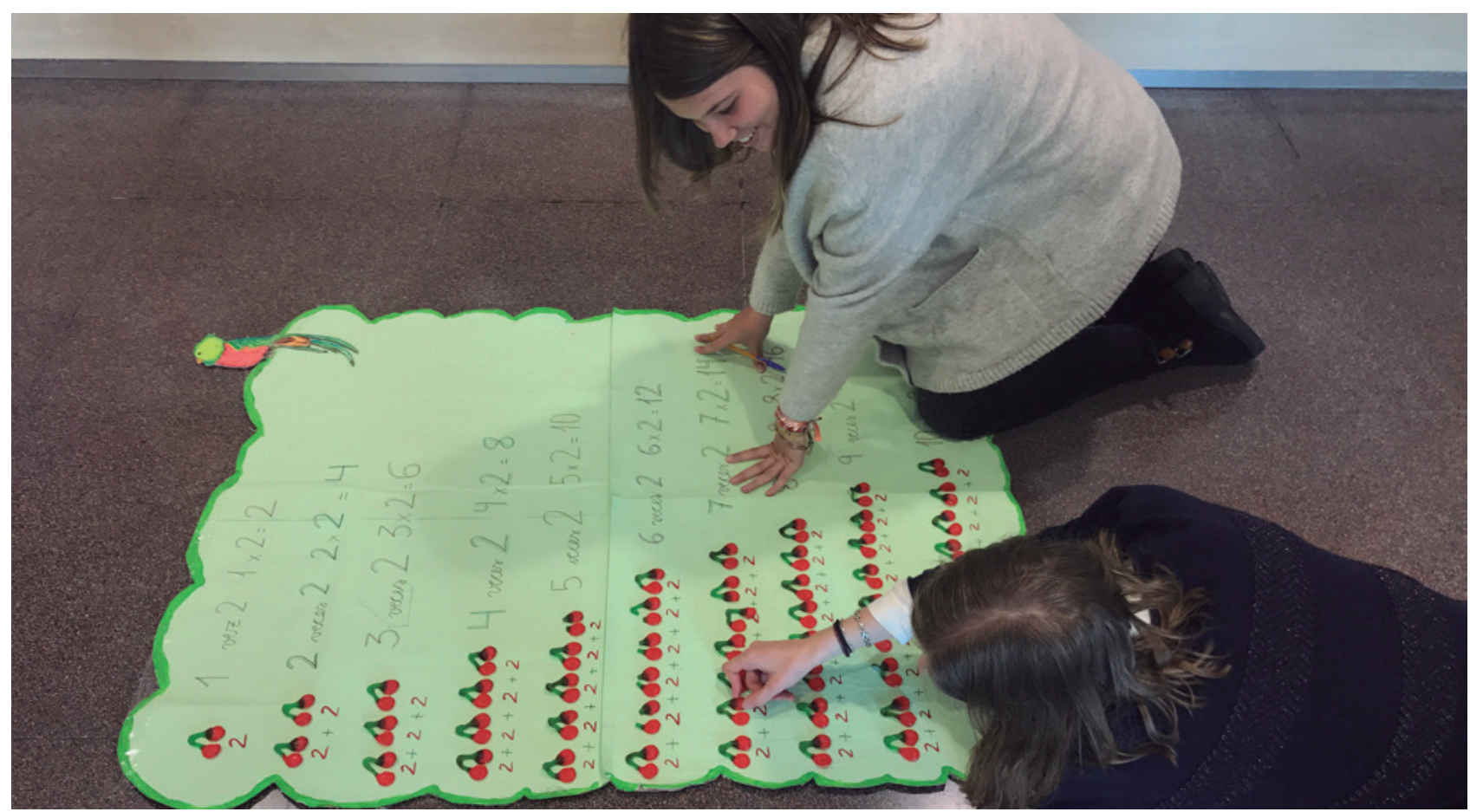

Canal Aurasma: matematicas.PyM
Por otro lado, y a nivel personal, el ApS para Guatemala nos ha ayudado a entender que la educación es una herramienta más poderosa de lo que podemos imaginar, pues con ella podemos cambiar el mundo.

Guatemala necesitaba nuestra ayuda, y no de cualquier manera. Se precisaba que colaboráramos en el progreso de los alumnos y en la formación de los maestros, ya que es en las manos de los niños donde reside un futuro mejor. Así, mientras que aportábamos un bien a los demás, tomábamos conciencia de la importancia de nuestra futura profe-

\section{DD) PARA SABER MÂS}

AlsinA, A. (2016). Diseño, gestión y evaluación de actividades matemáticas competenciales en el aula. Revista Epsilon, 33, 7-29.

Fernández Bravo, J. A. (2007a). La enseñanza de la multiplicación aritmética: una barrera epistemológica. Revista Iberoamericana de Educación, 43, 119-130.

Fernández Bravo, J. A. (2007b). Metodología didáctica para la enseñanza de la matemática: variables facilitadores del aprendizaje. En J. A. Fernández (Coord.), Aprender matemáticas. Metodología y modelos europeos (pp. 9-26). Madrid: MEC.

Frabetti, C. (2002). El monstruo del laberinto. En C. Frabetti (ed.), Malditas Matemáticas, 3rd ed. Madrid: Alfaguara juvenil.

SAntAOlalla, E. (2011). jMarchando una de matemáticas! Revista Padres y Maestros, 341, 10-13. sión y de lo esencial de la formación en el ámbito educativo.

Finalmente, despedimos el proyecto. No obstante, no es un adiós, sino un hasta pronto. Por un lado, ya hemos entregado los materiales a la Fundación del Valle, que se encargará de hacerlos llegar a Guatemala. Por otro lado, aquí en España, vamos a presentar un póster del proyecto en el VII Congreso Nacional y III Internacional de Aprendizaje-Servicio Universitario que tendrá lugar en Sevilla en octubre de 2017. Ha sido una experiencia muy enriquecedora y esperamos recibir pronto noticias de Guatemala.

Si quieres revivir nuestro cierre del proyecto, descárgate la aplicación Aurasma, accede al canal que se expone bajo la imagen y escanea la foto •

\section{(9) \\ Aprendizaje servicio; multiplicación; matemáticas; materiales y recursos; educación; emociones; Guatemala; enseñanza basada en problema (EBP).}

Este artículo fue solicitado por PADRES Y MAESTROS en marzo de 2017, revisado y aceptado en junio de 2017. 